\title{
An Infrastructure for Rule-Driven Negotiating Software Agents*
}

\author{
Morad Benyoucef, Hakim Alj and Rudolf K. Keller \\ Département d'informatique et de recherche opérationnelle \\ Université de Montréal, C.P. 6128 Succursale Centre-ville Montréal, Québec, H3C 3J7, Canada \\ \{benyouce,alj,keller\}@IRO.UMontreal.ca
}

\begin{abstract}
Most strategy-enabled automated negotiation platforms use hard-coded, predefined, and non-adaptive negotiation strategies. This is evidently insufficient in regard to the ambitions of automated negotiations research. In this paper, we introduce INSULA, an infrastructure for representing, managing, and exploring negotiation strategies that is versatile, easy to use, and that encompasses a wide spectrum of negotiation types. We treat negotiation strategies as declarative knowledge, and thus we represent them as if-then rules which software agents can exploit using an inference engine. The first part of the paper examines negotiation strategies and the possibility of using a rule-based approach to represent them. The second part details the architecture of INSULA, and some of the ongoing experimentations.
\end{abstract}

\section{Introduction}

The recent interest in autonomous interacting software agents and their potential application in areas such as ecommerce have given increased importance to automated negotiations [1]. We talk of automated negotiations when the negotiating function is performed by (networked) computers. A distinction should be made between fully automated negotiations, which require that all parties involved be software agents, and semi-automated negotiations, which involve humans negotiating with software agents. Manual negotiations, on the other hand, refer to processes in which all parties are human. Reeves et al. argue that contracting can benefit substantially from automation. They define contracting as a three stage process: (1) discovery, where the agents find potential contracting partners; (2) negotiation, where contract terms are determined through a communication process; and (3) execution of the contract [2]. Negotiating software agents can be mobile, i.e., move between different computers or reside on one computer. They also may have learning capabilities or base their actions on pre-defined rules of behavior [3]. Finally, they can be involved in competitive negotiations, meaning that they are adversaries with conflicting interests. They can, on the other hand, be involved in cooperative negotiations, and in this case, they all aim at satisfying the same interest.

Negotiation is a form of interaction defined in terms of protocols and strategies. The protocols comprise the rules (i.e., the valid actions) of the game. For a given protocol, a participant uses a rational strategy (i.e., a plan of action) to maximize her utility [4]. Most automated negotiation platforms use hard-coded, predefined, and non-adaptive negotiation strategies. This is evidently insufficient in regard to the ambitions of automated negotiations research.

We aim to provide an infrastructure for representing, managing, and exploring negotiation strategies. To this end, we designed a system called INSULA (INfraStructure for rULe-driven negotiating software Agents) that is versatile, easy to use, and that encompasses a wide spectrum of negotiation types such as multi-attribute, multi-product, multi-copy, and combined negotiations [5]. We treat negotiation strategies as declarative knowledge, and thus we represent them as ifthen rules which software agents are able to exploit using an inference engine. Rules are declarative statements that drive activity in a software application by describing the action(s) to take when a specified set of conditions is met.

Section 2 of the paper introduces negotiation strategies and reviews some projects that implement them. Next, we discuss the rule-based approach for representing these strategies. We then detail the architecture of INSULA, and discuss some of our ongoing experimentations. A short conclusion wraps up the paper.

\section{Negotiation Strategies}

Jennings et al. identify the following topics in automated negotiations [6]: (1) the negotiation protocols which cover the types of participants, the negotiation states, the events that cause negotiation states to change, and the valid actions; (2) the negotiation objects which

\footnotetext{
* This research is supported by Bell Canada, BCE Emergis, NSERC (National Sciences and Engineering Research Council of Canada), and CIRANO (Centre Interuniversitaire de Recherche en ANalyse des Organisations).
} 
define the range of issues over which agreement must be reached and the operations that can be performed on the issues; and (3) the decision making models which are used by the agents to achieve their objectives. The success of an agent is determined by the effectiveness of its decision making model.

According to Wong et al. [7], most current ecommerce systems use predefined and non-adaptive negotiation strategies (i.e., decision making models) in the generation of offers and counteroffers during the course of the negotiation. Commercial auction sites still require that users manage their own strategies over an extended period of time. Proxy bidding (a simple agent using a predefined strategy) is however offered on some auction sites to relieve the user from this task. The buying (selling) agents in the well-known KASBAH system [8], for instance, can choose between three predefined negotiation strategies: anxious, cool-headed and frugal, corresponding to linear, quadratic, and exponential functions, respectively, for increasing (or decreasing) their bid (or asking price) for an item over time.

Negotiating software agents face tough decisions such as whether or not to accept an offer, whether or not to make a counteroffer, how much to bid, etc. Evidently, those decisions should benefit from all the information that is available. The decisions may be even harder to take as the information is constantly changing and highly uncertain (other participants come and leave, prices keep on changing, etc.) According to Nwana et al., few negotiation strategies take into consideration the time aspects, and the fact that agents' goals, beliefs, intentions, etc. change with time [9]. Some other information that is useful to the agent is the valuation (i.e., the market price) of the item at stake, which might help avoid the winner's curse. Successful strategies should take into account the strategies of the opponents. Finally, the reputation of the opponents can be helpful in designing a winning strategy.

Various AI based techniques are used to provide adaptive behavior in the negotiation process. These techniques include logic, case-based reasoning (CBR), constraint-directed search, etc. [9]. The use of CBR, for instance, is justified by the fact that good negotiation skills in humans seem to come from experience [7].

The most cited strategy-enabled infrastructure for automated negotiations is KASBAH [8], a multi-agent classified ad system centered on the negotiation phase of an e-commerce transaction. The user (seller or buyer) of this system creates an agent, gives it instructions and sends it to a centralized agent marketplace. At the time of its creation, the user (a seller in this case) sets the desired price, the desired time to sell, the lowest acceptable price and the negotiation strategy. The negotiation strategy for sellers is based on the following rule: offer the item at the desired price, if there is no buyer, lower the asking price to generate more interest. At the desired date to sell, the asking price should be equal to the lowest acceptable price. The owner of a buyer (seller) agent can choose between three predefined strategies for increasing (decreasing) their bid (ask price) for an item over time.

Another well-known infrastructure is AuctionBot [10]. It is a configurable auction server that supports many auction types. Hu et al. designed an agent server [11] that works on the user's behalf by submitting bids to AuctionBot. The users specify the names of the auctions in which they want to participate, the initial amounts of the goods, and the bidding strategies they prefer. The agents bid until the auction closes, and then report the results back to the users. The agent server runs three types of agents: (1) competitive agents that always bid their true reservation prices; (2) price-modeling agents that base their next bid on the history data of clearing prices; and (3) bidder-modeling agents that model the actions of other agents. The strategies are hard-coded into the agents.

The FM97.6 system, part of the Fishmarket project [12], is yet another test-bed for electronic auctions which provides support to software agent developers in defining, activating, and evaluating experimental scenarios (i.e., tournaments). The tournaments are based on a downward bidding protocol (a variation of a Dutch auction). Trading (buyer and seller) heterogeneous (human and software) agents of arbitrary complexity participate in these tournaments and are evaluated according to their market performance. Agents are provided with strategies based on two different approaches: (1) a pragmatic approach that provides agents with heuristic guidelines, and (2) a formal approach based on possibilistic decision theory.

The proxy negotiation server of Su et al. [13], finally, lets the consumer register by giving a description of the items she wants, her preferences, and a negotiation strategy. The server then looks for a supplier that matches the consumer. When it finds one, it starts a bargaining type negotiation, using the negotiation strategy supplied by the consumer, until eventually a deal is reached. A declarative approach is used to represent the negotiation strategies.

\section{Declarative Representation of Business Rules and Negotiation Strategies}

Two approaches inspired us in choosing a mechanism for representing negotiation strategy knowledge.

The first one is the work of Grosof et al. at the IBM T. J. Watson laboratory [14]. Their research is concerned with the representation of business rules in contracts. It relies on the fact that many contract terms involve conditional relationships, and can conveniently be expressed as business rules. An example of such a rule is: (Rule A:) if buyer returns the purchased good for any reason, within 30 days, then the purchase amount, minus a 10\% restocking fee, will be refunded [14]. 
A shared language is sought, with which agents can reach a common understanding of rules in contracts. The rules should be easily modifiable, communicable, and executable by the agents [14]. There are several approaches for representing rules: (1) if-then code constructs within an imperative language such as $\mathrm{C}++$ or Java, (2) declarative languages such as Prolog, (3) SQL views, (4) event-condition-action rules / "active rules" / "triggers", (5) production rules, and (6) Knowledge Interchange Format (KIF). None of the approaches cited above satisfies completely the requirements stated by Grosof et al., and so, a new approach called Courteous Logic (CL) was devised. CL is an extension of ordinary logic achieved by adding the possibility to express prioritized conflict handling as rules. The following example taken from [15] gives an idea of the syntax used. <leadTimeRule1>

modificationNotice(?Buyer, ?Seller, ?Flight, 14days) $\leftarrow$ PreferedCustomerOf(?Buyer, ?Seller).

The rule states: "a buyer can modify the departure time up until 14 days before scheduled departure if the buyer is a preferred customer."

The second approach is the work of $\mathrm{Su}$ et al. at the University of Florida [13]. Contrary to some existing approaches in which negotiation strategies are hard-coded in negotiation agent programs, they use a high-level rule specification language and GUI tools to allow human negotiation experts to edit negotiation strategies at runtime [13]. Each strategy is expressed in terms of an Event-Trigger-Rule (ETR), which specifies the condition to be checked and the actions to be taken by the negotiation server. Rules are activated upon the occurrence of specific events during the negotiation. Instead of a rule engine, an ETR server is used to manage events and trigger rules that are relevant to the posted event. The following ETR example is taken from [13]:

\section{TriggerEvent}

SR1:

$$
\text { SupplierComputer_Systemdelivery_day }
$$

Condition: getLowBound("delivery_day") > 10

Action: downLowBound("delivery_day", 2);

Alternative: unResolvable("delivery_day cannot be satisfied");

The rules states: "if the closest possible delivery date is more than 10 days away from the current date, then shorten the delivery time by two. Otherwise, human intervention is required."

Rules have two main advantages over other software specification approaches and programming languages. First, they are at a relatively high level of abstraction, and are closer to human understanding, especially by business domain experts who are typically non-programmers. Second, they are relatively easy to modify dynamically [15]. New behavior can be specified by simply adding rules, without needing to modify the previous ones.
Furthermore, rules can be augmented with procedural attachments (i.e., procedural calls) so that they have an effect beyond pure-belief inferencing.

\section{Architecture of INSULA}

As seen in Figure 1, INSULA is composed of three main components. The first component, the User Interface, has two parts. The Agent Control \& Monitoring part is used to provide all the information that is required of any participant in a negotiation be it a human or a software agent (address of the negotiation server, login, password, etc.). It is also used for launching agents once they have been instantiated by the Agent Factory. And most importantly, it is used for controlling and monitoring the actions of the agents. The second part of the user interface is the Rule Entry \& Editing part, which is used to enter, and eventually edit rules. The Rule Processing component consists of a Rule Repository and an Inference Engine. The Rule Repository is used to store the rules. We support three classes of rules. (1) The working rules embody the basic behavior of agents, depending on the type of negotiation involved. An example of a working rule is: if the reserve-price is met then drop out of the negotiation. Note that this rule is specific to an English auction, and it instructs the agent to quit the auction when its reserveprice is met. (2) The strategy rules are meant to maximize the utility of an agent. An example would be: if the opponents are cautious then be less aggressive in your bidding. Here again, the rule applies to an English auction, and it is obviously more complex than a working rule since it is based on information about the opponents and on an evaluation of how cautious they are. (3) The coordination rules are used in case the user instantiates and launches many agents and there is a need to coordinate their actions. The user might for instance need to negotiate one single item on different negotiation servers and only take the least expensive one (if the price is the only negotiable issue). An obvious coordination rule would be: if another INSULA agent has made a commitment on the item then do not bid on it. The Inference Engine is used to exploit the rules in order to determine the action (actions) to take (whether to bid or not, how much to bid, what offer or counteroffer to make, whether to call for human intervention, etc.). The Agent Instantiation component is used to instantiate the agents automatically. Agents are created by the Agent Factory according to the negotiation type practiced on the negotiation server at hand. For that reason, a description of the negotiation is downloaded from the server into a Negotiation Description repository, which will be used by the Agent Factory in the instantiation process.

In the first implementation of INSULA, the agents operate in repeated cycles we call pulses [5]. A pulse is made of three phases: get information, think, and act. The 
first phase consists of querying the server for information about the negotiation (the highest bid, the remaining time, the identity of the highest bidder, etc.). This phase was introduced because the currently used negotiation server functions only in pull mode. We intend to adjust this model once we have a server that offers the push mode. The think phase of the pulse consists of applying the rules. This will be carried out by the inference engine, which will identify as a result the action to take. The final phase of the pulse is the action itself.

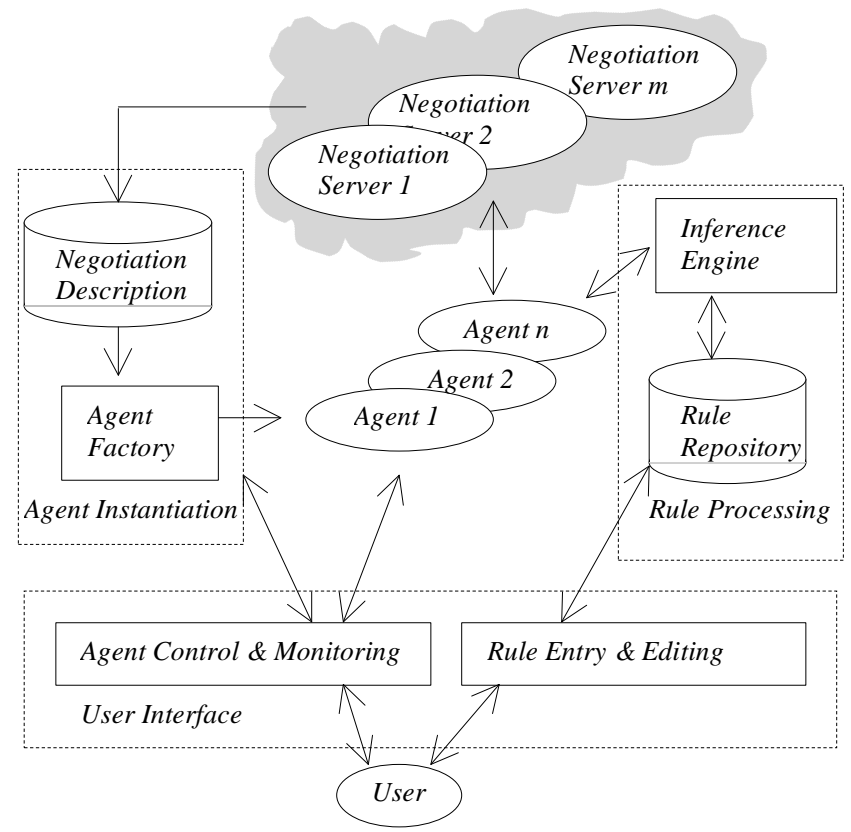

Figure 1. Architecture of INSULA

The implementation of INSULA is based on ILOG's JRules [16]. JRules is a rule engine that combines rulebased techniques with object-oriented programming. It gives a Java application the possibility to exploit a knowledge base. GENESIS [17] is the negotiation server we use in our experiments. It allows the user to create any type of negotiation, and to submit orders and get responses by exchanging XML documents. We created several English auctions on GENESIS and had our software agents connect and participate in them. The information that is made available to the bidding agents via the available methods of the API is still quite basic, GENESIS being a product of ongoing work. We have implemented rules based on the information available, and we intend to implement more complex ones as soon as GENESIS provides the means for it.

\section{Experimentations}

We are currently exploring the following scenarios:

- A one agent, one negotiation scenario where one software agent is created and made to participate in a negotiation. Human agents participate in the same negotiation as opponents to the software agent.

- An $n$ agents, one negotiation scenario where several software agents are created and made to participate in the same negotiation. The agents can be of different types and dispose of different rules. We observe the events of the negotiation, which enables us to evaluate the effectiveness of given negotiation strategies and also to compare different strategies.

- An $n$ agents, $m$ negotiations scenario where many agents collaborate in negotiating a package of items using strategies that apply to the individual negotiations and strategies that deal with their coordination. This latter scenario is a combined negotiation case [5].

For our first experimentations we have implemented two types of agents: reservation-price agents and progressive-price agents. Moreover, we built variations of these by making agents that are fully autonomous and others that are semi-autonomous. In the following, we present a summary of these experimentations.

\section{Simple reservation-price agent}

A reservation-price agent is given a reserve-price (an amount not to be exceeded) and an increment (an amount to be added to the current bid to make the new bid). The agent can be fully autonomous and thus, whenever there is a new bid, the agent bids that amount plus the increment. When the reserve-price is met, the agent drops out of the auction. The reserve-price agent can also be semi-autonomous. In this case it acts as an autonomous agent except in certain situations where it asks for user intervention. Figure 2 shows one of the rules given to a simple reservation-price agent.

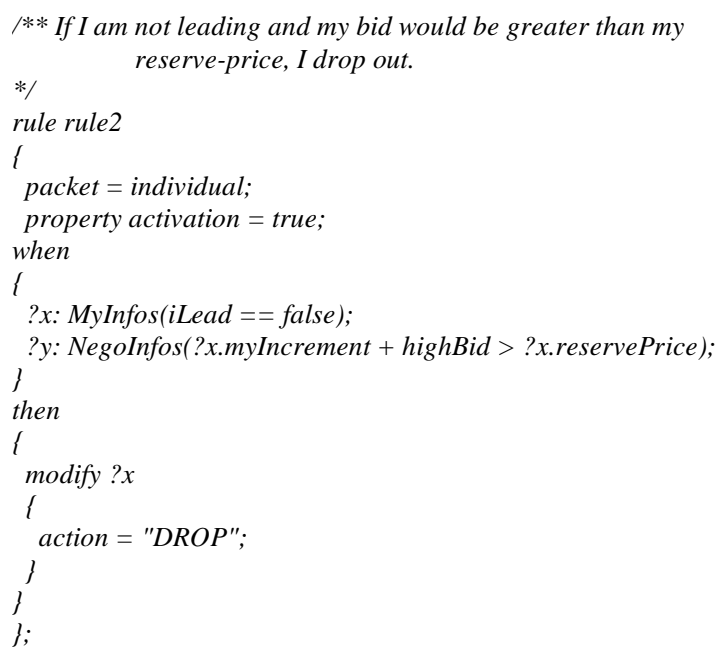

Figure 2. A simple reservation-price rule

\section{Complex reservation-price agent}

Here again we use a reservation-price agent but we augment its knowledge with more elaborate rules. These more elaborate rules are based on information that the agent gathers from the negotiation server such as the 
frequency of bids, the average increment, the identities of the bidders, their profiles, etc. Figure 3 shows one of the rules given to a complex reservation-price agent.

\section{Semi-autonomous progressive-price agent}

The agent is given an interval (a lower bound and an upper bound). Whenever the highest bid is within the interval, the agent queries the user for more information. The user can instruct the agents to drop out of the auction, to wait, or she can decide to give it a new interval. If the highest bid plus the increment is less than the lower bound, then the agent bids the highest bid plus the increment. If the highest bid is greater than the upper bound, then the agent drops out of the auction. Figure 4 shows one of the rules given to a semi-autonomous progressive-price agent.

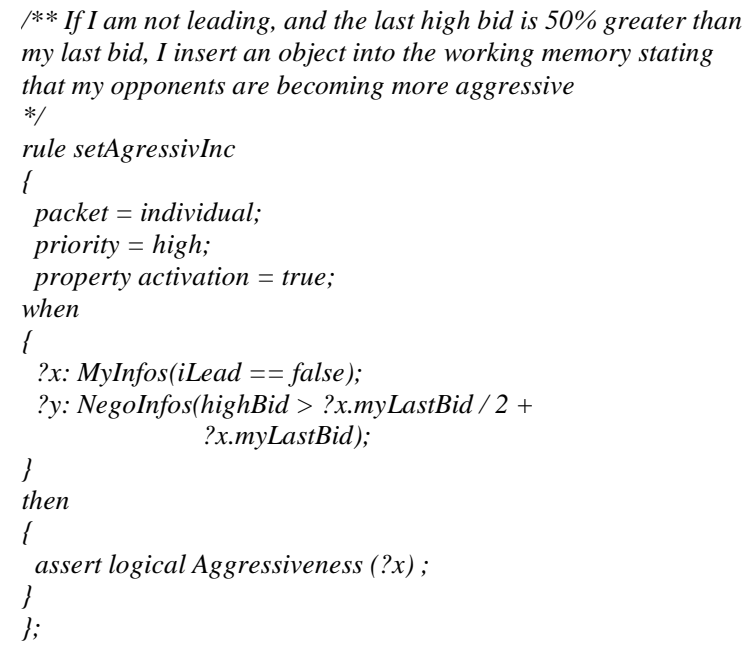

\section{Figure 3. A complex reservation-price rule}

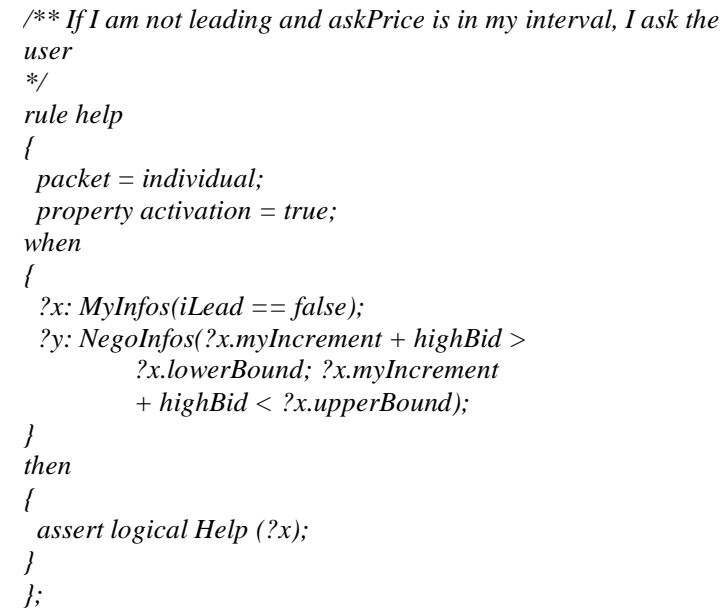

Figure 4. A semi-autonomous progressive-price rule

\section{Conclusion}

The paper introduced INSULA, an agent-based strategyenabled negotiation infrastructure that uses if-then rules to represent negotiation strategies. The reasons for this representation are: rules are at a high level of abstraction, are close to human understanding, are easy to modify before and during the negotiation process, can be augmented with procedural attachments, and can be exploited by the agents using off-the shelf rule engines. A proof-of-concept implementation, together with a set of experimentations were also presented. Validation of this work will be complemented with an in-depth analysis of its strengths and weaknesses. Finally, we see INSULA as a valuable tool in conducting experimentations of known and newly designed negotiation strategies (not only auctions strategies).

\section{References}

[1] D. Zen and K. Sycara. Bayesian Learning in Negotiation. Intl. Jrnl of Human-Computer Studies, 48, pp. 125-141, 1998.

[2] D. M. Reeves, B. N. Grosof, M. P. Wellman, and H.Y. Chan. Automated negotiations from formal contract descriptions. In IBM/IAC workshop on Internet-based Negotiation Technologies, March 1999.

[3] G. Lo and G.E. Kersten. Negotiation in Electronic Commerce: Integrating Negotiation Support and Software Agent Technology. In Proceedings (CD-ROM) of the $29^{\text {th }}$ Atlantic Schools of Business Conference, Halifax, NS, 1999.

[4] R. H. Guttman, A. G. Moukas, and P. Maes. Agent-mediated ecommerce: A survey. Knowledge Engineering 13(3), 1998.

[5] M. Benyoucef, H. Alj, M. Vézeau, and R. K. Keller. Combined Negotiations in E-Commerce: Concepts and Architecture. Electronic Commerce Research Journal, 2, 2001. Special issue on Theory and Application of Electronic Market Design. Baltzer Sc. Pub. To appear. [6] N. R. Jennings, P. Faratin, A. R. Lomuscio, S. Parsons, C. Sierra, and M. Wooldridge. Automated negotiation: Prospects, methods and challenges. International Journal of Group Decision and Negotiation, 10(2), 2001. To appear.

[7] W. Y. Wong, D. M. Zhang, M. Kara-Ali. Negotiating with Experience. In Proc. of KBEM'00. Austin, Texas. July 2000.

[8] A. Chavez and P. Maes. KASBAH: An agent marketplace for buying and selling goods. In Conf. on Practical Application of Intelligent Agents and Multi-agent Technology, April 1996.

[9] H. S. Nwana, L. Lee, and N. R. Jennings. Co-ordination in software agent systems. BT Technology Journal, 14(4):79-89, Oct 1996.

[10] P. R. Wurman, M. P. Wellman, and W. E. Walsh. The Michigan Internet AuctionBot: A configurable auction server for human and software agents. In Second Intl. Conf. on Autonomous Agents, 301-308, Minneapolis, MN, May 1998.

[11] J. Hu, D. Reeves, and H. Wong. Agent service for online auctions. In AI in E-Commerce Workshop, Menlo Park, 1999.

[12] J. A. Rodriguez-Aguilar, F. J. Martin, P. Noriega, P. Garcia, and C. Sierra. Towards a test-bed for trading agents in electronic auction markets. AI Communications, 11(1):5-19, 1998.

[13] Y. Su, C. Huang, and J. Hammer. A Replicable Web-based Negotiation Server for E-Commerce. In Proc (CD_ROM) of 33rd Intl Conf on System Sciences, Hawaii, 2000.

[14] B. N. Grosof, Y. Labrou, and H. Y. Chan. A declarative approach to business rules in contracts: Courteous logic programs in XML. In 1st Conference on Electronic Commerce, Denver, Colorado, Nov 1999.

[15] D. M. Reeves, B. N. Grosof, M. P. Wellman, and H. Chan. Toward a declarative language for negotiating executable contracts. In AI in ECommerce Workshop, Menlo Park, 1999.

[16] ILOG JRules. http://www.ilog.com/products/jrules/.

[17] M. Benyoucef, R. K. Keller, S. Lamouroux, J. Robert, and V. Trussart. Towards a generic e-negotiation platform. In Sixth Intl Conf on Re-Technologies for Information Systems, p 95-109, Zurich, Switzerland, Feb 2000. Austrian Computer Society. 